\title{
Maturation Patterns of EEG Basic Waves of Healthy Infants under Twelve-months of Age
}

\author{
Takashi Mizuno, Norio Yamauchi, Ayuko Watanabe, \\ Mayuki Komatsushiro, Tokuro Takagi, Kazuie Iinuma \\ and Tsuneo Arakawa \\ Department of Pediatrics, Tohoku University School of \\ Medicine, Sendai
}

Mizuno, T., Yamauchi, N., Watanabe, A., Komatsushiro, M., Takagr, T., IIndma, K. and Arakawa, Ts. Maturation Patterns of EEG Basic Waves of Healthy Infants under Twelve-months of Age. Tohoku J. exp. Med., 1970, 102(1), 91-98. - A simplified method for an evaluation of maturation patterns of the EEG waves of healthy infants under 12 months of age was reported. The EEG basic waves were divided into 10 bands according to frequency ranges ( $\mathrm{I}-\mathrm{X}$ bands). An averaged integrated voltage was calculated at each of the frequency bands (I-E\%, II $-\mathrm{E} \%, \ldots . \mathrm{X}-\mathrm{E} \%)$. When ' $\mathrm{R}$ ' $=(\mathrm{E} \%$ of $\mathrm{VIII}+\mathrm{E} \%$ of $\mathrm{IX}) /(\mathrm{E} \%$ of $\mathrm{II}+\mathrm{E} \%$ of III $)$ was claculated and plotted against the age in months, a linear figure was obtained which demonstrated a maturation pattern of the EEG basic waves of infants with advance in age. __EEG, frequency analysis, maturation pattern of EEG.

The present study was attempted to establish a simplified method for an evaluation of maturation patterns of the EEG basic waves of infants under 12 months of age, by using a frequency analyzer so specially adapted that the basic waves of EEG were divided into 10 bands according to the frequency range (Arakawa et al., 1969(a); Arakawa et al., 1969(b)).

\section{Methods and Materials}

The infants subjected to the present study consisted of 10 infants who were selected as being 'clinically' healthy on the basis of the following clinical criteria: They were born to healthy parents after an uneventful pregnancy and delivery. Neonatal history revealed none of asphyxia, cyanosis, convulsion, severe jaundice and other abnormalities. Five infants (Cases 1-5) were breast-fed, four (Cases 6-9) were artificially fed, and one (Case 10) was placed on a mixed feeding. Weaning was done uneventfully when the infants were 5-6 months old. Physical examination, measurement of body weight, height, circumference of the head and chest were performed monthly at a Well-Baby-Clinic of our Hospital and those whose body weight deviated from the zone of the mean value $\pm^{2}$ standard deviations for the corresponding age (Report from the Department of Welfare, Japan, 1960) were excluded.

Estimation of an integrated voltage at each of the EEG frequency bands

The EEG recording was performed in the same way as described in the preceding papers of ours (Arakawa et al., 1969(a); Arakawa et al., 1969(b)). A 12 channel electroencepha-

Received for publication, June 11, 1970. 
TABLE 1. Physical development of Case 1 (F.Y.\&)

\begin{tabular}{|c|c|c|c|c|c|}
\hline $\begin{array}{l}\text { Age } \\
\text { (months } \\
\text { and } \\
\text { days) }\end{array}$ & $\begin{array}{l}\text { Body } \\
\text { weight } \\
\text { (g) }\end{array}$ & $\begin{array}{l}\text { Height } \\
\text { (cm) }\end{array}$ & $\begin{array}{c}\text { Circumference } \\
\text { of head } \\
(\mathrm{cm})\end{array}$ & $\begin{array}{c}\text { Circumference } \\
\text { of chest } \\
(\mathrm{cm})\end{array}$ & Diet \\
\hline $\begin{array}{r}\text { Birth } \\
1-16 \\
4-16 \\
\\
5-27 \\
7-7 \\
8-4 \\
9-2 \\
10-15 \\
11-11 \\
12-10\end{array}$ & $\begin{array}{l}3,510 \\
5,530 \\
(1129) * \\
8,000 \\
8,570 \\
9,040 \\
9,180 \\
9,560 \\
9,500 \\
9,860 \\
10,220 \\
(120 \%) *\end{array}$ & $\begin{array}{c}56.0 \\
(103 \%) * \\
66.0 \\
67.5 \\
71.0 \\
71.0 \\
73.5 \\
75.0 \\
77.2 \\
(106 \%) *\end{array}$ & $\begin{array}{c}37.8 \\
(102 \%) * \\
42.5 \\
43.7 \\
45.0 \\
45.5 \\
45.5 \\
45.7 \\
46.7 \\
46.0 \\
(102 \%) *\end{array}$ & $\begin{array}{c}36.5 \\
(98 \%) * \\
42.2 \\
43.0 \\
45.0 \\
47.0 \\
45.0 \\
46.5 \\
46.0 \\
45.5 \\
(101 \%) *\end{array}$ & $\begin{array}{l}\text { Mother's milk } \\
+ \text { Rice-gruel, soy bean } \\
\text { soup, vegetables } \\
\text { + Rice, butter }\end{array}$ \\
\hline
\end{tabular}

*( ) shows \% of the standard values reported from the Department of Welfare, Japan, in 1960.

Note. Holding up the head, 3 months; sitting, $51 / 2$ months; standing, 7 months; walking, 11 months.

TABLE 2. Physical development of Case 2 (O.N.\&)

\begin{tabular}{|c|c|c|c|c|c|}
\hline $\begin{array}{l}\text { Age } \\
\text { (Months } \\
\text { and } \\
\text { days) }\end{array}$ & $\begin{array}{c}\text { Body } \\
\text { weight } \\
\text { (g) }\end{array}$ & $\begin{array}{c}\text { Height } \\
(\mathrm{cm})\end{array}$ & $\begin{array}{l}\text { Circumference } \\
\text { of head } \\
(\mathrm{cm})\end{array}$ & $\begin{array}{l}\text { Circumference } \\
\text { of chest } \\
(\mathrm{cm})\end{array}$ & Diet \\
\hline $\begin{array}{l}\text { Birth } \\
1-4 \\
2-2 \\
3-6 \\
4-3 \\
5-1 \\
5-28 \\
6-26 \\
8-2 \\
8-27 \\
9-27 \\
12-6\end{array}$ & $\begin{array}{l}3,220 \\
4,500 \\
(100 \%)^{*} \\
5,520 \\
6,400 \\
7,100 \\
7,780 \\
8,050 \\
8,170 \\
9,170 \\
9,740 \\
9,850 \\
10,400 \\
(122 \%)^{*}\end{array}$ & $\begin{array}{l}53.5 \\
(989) * \\
57.0 \\
60.2 \\
63.0 \\
65.5 \\
67.5 \\
70.2 \\
72.0 \\
72.5 \\
71.8 \\
75.5 \\
(98 \%) *\end{array}$ & $\begin{array}{c}35.5 \\
(93 \%) * \\
38.0 \\
40.0 \\
41.0 \\
42.0 \\
43.0 \\
43.5 \\
44.5 \\
44.5 \\
45.0 \\
46.5 \\
(103 \%) *\end{array}$ & $\begin{array}{l}37.5 \\
(100 \%) * \\
39.0 \\
42.5 \\
45.0 \\
44.0 \\
46.5 \\
46.5 \\
47.0 \\
45.0 \\
49.0 \\
(109 \%) *\end{array}$ & $\begin{array}{l}\text { +Rice-gruel, egg } \\
\text {-yolk, vegetables } \\
\text { +Fish }\end{array}$ \\
\hline
\end{tabular}

( )*, of. Table 1

Note. Holding up the head, 3 months; sitting, 5 months; standing, 6 months.

lograph (Toshiba-St-2477-A) was used and disc electrodes were placed on the scalp according to the 10/20 international system. The EEG at the rest with closed eyes from the right occipital area was recorded on a magnetic tape using a 4 channel data recorder.

The EEG basic waves were divided into 10 bands according to frequency ranges by means of a frequency analyzer specially adapted as follows: I band, $2-2.4 \mathrm{c} / \mathrm{s}$; II band, $2.4-2.88 \mathrm{c} / \mathrm{s}$; III band, $2.88-3.46 \mathrm{c} / \mathrm{s}$; IV band, $3.46-4.15 \mathrm{c} / \mathrm{s} ; \mathrm{V}$ band, $4.15-4.98 \mathrm{c} / \mathrm{s}$; VI band, $4.98-5.97 \mathrm{c} / \mathrm{s}$; VII band, $5.97-7.17 \mathrm{c} / \mathrm{s} ;$ VIII band, $7.17-8.60 \mathrm{c} / \mathrm{s}$; IX band, $8.60-10.3 \mathrm{c} / \mathrm{s}$, and $\mathrm{X}$ band, $10.3-12.4 \mathrm{c} / \mathrm{s}$.

An integrated voltage at each band was an average for 12 epochs of the 5 sec. period 
Table 3. Physical development of Case 3 (O.Y. $\delta$ )

\begin{tabular}{|c|c|c|c|c|c|}
\hline $\begin{array}{l}\text { Age } \\
\text { (months } \\
\text { and } \\
\text { days) }\end{array}$ & $\begin{array}{l}\text { Body } \\
\text { weight } \\
\text { (g) }\end{array}$ & $\begin{array}{l}\text { Height } \\
(\mathrm{cm})\end{array}$ & $\begin{array}{c}\text { Circumference } \\
\text { of head } \\
(\mathrm{cm})\end{array}$ & $\begin{array}{l}\text { Circumference } \\
\text { of chest } \\
\text { (cm) }\end{array}$ & Diet \\
\hline Birth & 2,820 & & & & Mother's milk \\
\hline $1-5$ & $\begin{array}{l}4,510 \\
(969) *\end{array}$ & $\begin{array}{c}54.7 \\
(98 \%) *\end{array}$ & $\begin{array}{l}38.0 \\
(100 \%) *\end{array}$ & $\begin{array}{c}36.5 \\
(93 \%) *\end{array}$ & \\
\hline $2-10$ & 5,600 & 57.5 & 39.5 & 40.5 & \\
\hline $3-7$ & 6,300 & 61.4 & 41.2 & 41.0 & \\
\hline 4- 4 & 7,000 & 65.6 & 42.0 & 45.0 & \\
\hline $5-10$ & $\begin{array}{l}7,430 \\
(100 \%)^{*}\end{array}$ & $\begin{array}{c}67.5 \\
(103 \%) *\end{array}$ & $\begin{array}{l}43.5 \\
(100 \%)^{*}\end{array}$ & $\begin{array}{l}45.5 \\
(105 \%) *\end{array}$ & \\
\hline \multicolumn{6}{|c|}{)$^{*}$, cf. Table 1} \\
\hline
\end{tabular}

Note. Holding up the head, 4 months.

TABLE 4. Physical development of Case 4 (K.K.古)

\begin{tabular}{|c|c|c|c|c|c|}
\hline $\begin{array}{c}\text { Age } \\
\text { (months } \\
\text { and } \\
\text { days) }\end{array}$ & $\begin{array}{l}\text { Body } \\
\text { weight } \\
\text { (g) }\end{array}$ & $\begin{array}{l}\text { Height } \\
\text { (cm) }\end{array}$ & $\begin{array}{c}\text { Circumference } \\
\text { of head } \\
\text { (cm) }\end{array}$ & $\begin{array}{l}\text { Cireumference } \\
\text { of chest } \\
\text { (cm) }\end{array}$ & Diet \\
\hline Birth & 3,080 & & & & Mother's milk \\
\hline $1-3$ & $\begin{array}{l}4,690 \\
(99 \%) *\end{array}$ & $\begin{array}{l}54.5 \\
(98 \%) *\end{array}$ & $\begin{array}{c}37.8 \\
(99 \%)^{*}\end{array}$ & $\begin{array}{c}36.0 \\
(97 \%) *\end{array}$ & \\
\hline $2-8$ & 5,770 & 57.7 & 39.0 & 41.0 & \\
\hline $3-5$ & 6,270 & 61.0 & 40.0 & 39.0 & \\
\hline $4-2$ & 7,290 & 61.7 & 42.0 & 43.0 & \\
\hline $5-0$ & 7,610 & 65.0 & 43.0 & 43.0 & + Rice-gruel, apples \\
\hline $5-27$ & 7,800 & 65.7 & 43.0 & 43.0 & and Egg-yolk \\
\hline $6-25$ & 8,600 & 67.9 & & $42 \cdot 2$ & \\
\hline $7-29$ & 8,610 & 70.0 & 44.0 & 43.5 & \\
\hline $9-24$ & 9,100 & 70.0 & 45.0 & 43.8 & + Fish and meat \\
\hline $11-0$ & 9,390 & 73.4 & 47.0 & 46.0 & \\
\hline $11-28$ & $\begin{array}{l}9,700 \\
(106 \%) *\end{array}$ & $\begin{array}{l}74.0 \\
(99 \%) *\end{array}$ & $\begin{array}{l}46.0 \\
(10096) *\end{array}$ & $\begin{array}{c}47.5 \\
(10306) *\end{array}$ & \\
\hline & 12000 & & & & \\
\hline
\end{tabular}

()$^{*}$, of. Table 1

Note. Holding up the head, 2 months; sitting, 5 months; standing, 11 months.

TaBle 5. Physical development of Case 5 (T.Y. \&)

\begin{tabular}{c|c|c|c|c|c}
\hline $\begin{array}{c}\text { Age } \\
\text { (months } \\
\text { and } \\
\text { days) }\end{array}$ & $\begin{array}{c}\text { Body } \\
\text { weight } \\
(\mathrm{g})\end{array}$ & $\begin{array}{c}\text { Height } \\
(\mathrm{cm})\end{array}$ & $\begin{array}{c}\text { Circumference } \\
\text { of head } \\
(\mathrm{cm})\end{array}$ & $\begin{array}{c}\text { Circumference } \\
\text { of chest } \\
(\mathrm{cm})\end{array}$ & Diet \\
\hline Birth & 3,600 & & & & Mother's milk \\
$1-15$ & 5,380 & 56.7 & 38.5 & 39.0 & \\
& $(114 \%) *$ & $(104 \%) *$ & $(104 \%) *$ & $(104 \%) *$ & + Soy bean soup, fruit \\
$3-9$ & 6,750 & 63.2 & 41.3 & 39.0 & + Cereals \\
$4-14$ & 7,480 & 65.1 & 41.5 & 44.5 & \\
$5-11$ & 7,810 & 67.5 & & 46.0 & \\
$6-9$ & 8,270 & 67.0 & 42.5 & 41.0 & \\
$7-15$ & 9,030 & & 45.5 & 44.0 & \\
$8-10$ & 9,310 & 71.0 & 45.7 & $(104 \%) *$ &
\end{tabular}

( $)^{*}$, ef. Table 1

Note. Holding up the head, 2 months; sitting, 4 months. 
TABLE 6. Physical development of Cases 6 (Y.Y. १)

\begin{tabular}{|c|c|c|c|c|c|}
\hline $\begin{array}{l}\text { Age } \\
\text { (months } \\
\text { and } \\
\text { days) }\end{array}$ & $\begin{array}{l}\text { Body } \\
\text { weight } \\
\text { (g) }\end{array}$ & $\begin{array}{c}\text { Height } \\
\text { (cm) }\end{array}$ & $\begin{array}{l}\text { Circumference } \\
\text { of head } \\
(\mathrm{cm})\end{array}$ & $\begin{array}{l}\text { Circumference } \\
\text { of chest } \\
(\mathrm{cm})\end{array}$ & Diet \\
\hline $\begin{array}{r}1-16 \\
\\
2-14 \\
3-11 \\
4-8 \\
5-13 \\
6-10 \\
7-8 \\
8-12 \\
10-9 \\
11-20\end{array}$ & $\begin{array}{l}4,580 \\
(10196) * \\
5,190 \\
5,780 \\
6,280 \\
6,520 \\
6,890 \\
6,660 \\
7,270 \\
7,500 \\
7,880 \\
(9396) *\end{array}$ & $\begin{array}{l}54.4 \\
(101 \%) * \\
57.3 \\
60.2 \\
64.5 \\
64.6 \\
65.0 \\
66.3 \\
69.3 \\
69.0 \\
71.6 \\
(1009) *\end{array}$ & $\begin{array}{l}38.5 \\
(103 \%)^{*} \\
40.2 \\
41.3 \\
41.5 \\
42.5 \\
43.0 \\
43.0 \\
44.5 \\
45.0 \\
44.0 \\
(99 \%)^{*}\end{array}$ & $\begin{array}{c}37.0 \\
(99 \%) * \\
37.5 \\
39.0 \\
41.7 \\
42.0 \\
43.0 \\
40.7 \\
42.5 \\
43.0 \\
44.5 \\
(1009) *\end{array}$ & $\begin{array}{l}+ \text { Soy bean soup, } \\
\text { rice gruel } \\
+ \text { Egg yolk } \\
+ \text { Rice, fish }\end{array}$ \\
\hline
\end{tabular}

)$^{*}$, ef. Table I

TABLE 7. Physical development of Case 7 (M.S. $\delta)$

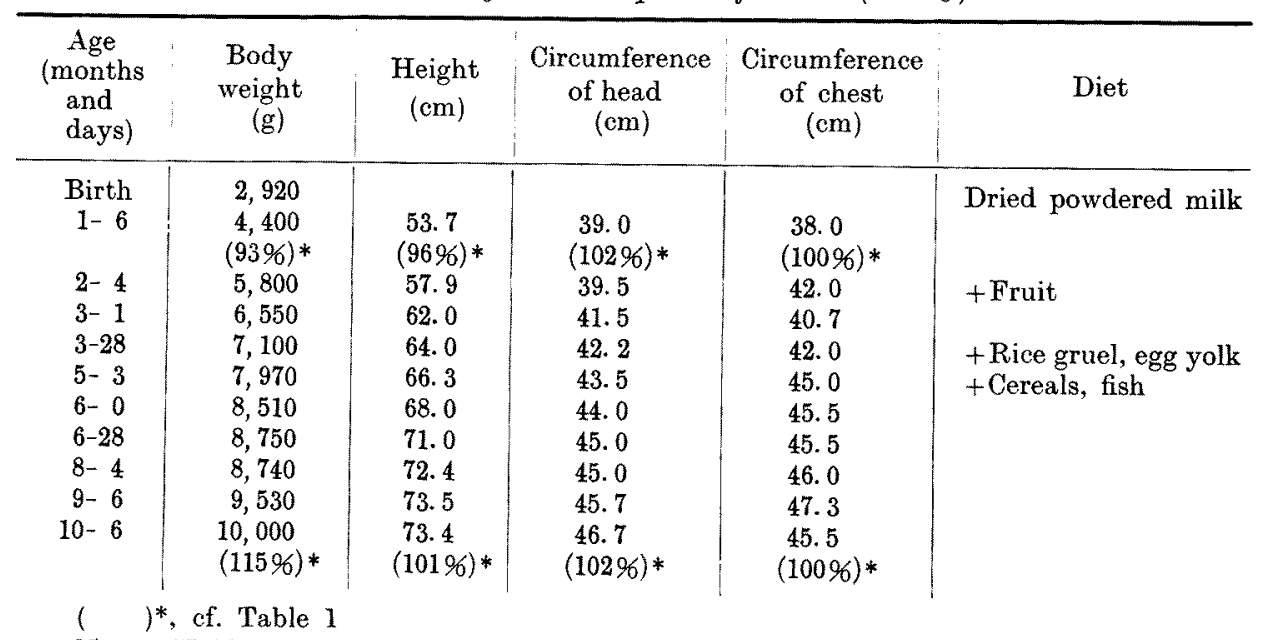

Note. Holding up the head, 3 months; standing, 9 months.

at each of the frequency bands. The averaged integrated voltage for the I band was calculated according to the following formula:

$$
E \%(I)=\frac{I^{2}}{I^{2}+I I^{2}+\cdots+X^{2}} \times 100
$$

\section{Results and Discussion}

Physical development

Measurements of body weight and height during the period of this study remained within a zone between +2 and -2 standard deviations as compared 
Table 8. Physical development of Case 8 (Y.K.\&)

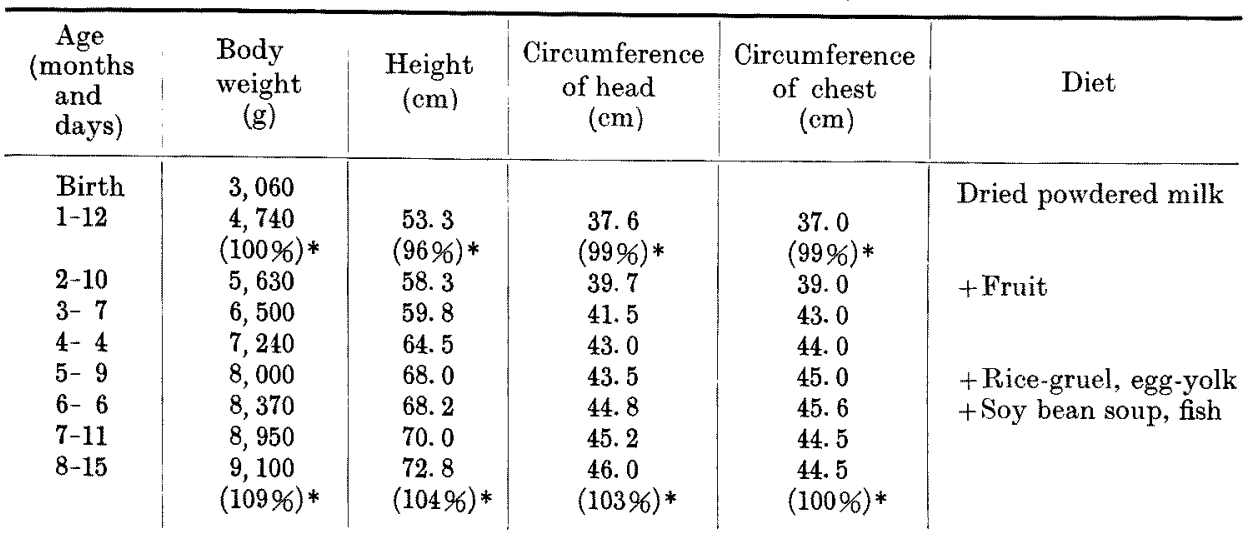

( $)^{*}$, of. Table 1

Note. Holding up the head, 2 months; sitting, 7 months.

Table 9. Physical development of Case 9 (K.C.\&)

\begin{tabular}{|c|c|c|c|c|c|}
\hline $\begin{array}{l}\text { Age } \\
\text { (months } \\
\text { and } \\
\text { days) }\end{array}$ & $\begin{array}{l}\text { Body } \\
\text { weight } \\
\text { (g) }\end{array}$ & $\begin{array}{l}\text { Height } \\
(\mathrm{cm})\end{array}$ & $\begin{array}{l}\text { Circumference } \\
\text { of head } \\
\text { (cm) }\end{array}$ & $\begin{array}{c}\text { Circumference } \\
\text { of chest } \\
(\mathrm{cm})\end{array}$ & Diet \\
\hline $\begin{array}{l}\text { Birth } \\
1-4\end{array}$ & $\begin{array}{c}3,340 \\
4,400 \\
(98 \%)^{*}\end{array}$ & $\begin{array}{c}5.40 \\
(99 \%) *\end{array}$ & $\begin{array}{c}36.0 \\
(97 \%) *\end{array}$ & $\begin{array}{c}37.0 \\
(99 \%) *\end{array}$ & Dried powdered milk \\
\hline $\begin{array}{l}2-9 \\
4-1\end{array}$ & $\begin{array}{c}5,950 \\
6,540 \\
(102 \%) *\end{array}$ & $\begin{array}{c}62.5 \\
64.7 \\
(1049) *\end{array}$ & $\begin{array}{l}39.5 \\
41.0 \\
(1009) *\end{array}$ & $\begin{array}{c}40.0 \\
41.0 \\
(99 \%)^{*}\end{array}$ & + Fruit, soy bean soup \\
\hline
\end{tabular}

( $)^{*}$, cf. Table 1

Note. Holding up the head, 4 months.

TABLE 10. Physical development of Case 10 (Y.N. $\hat{\text { ) }})$

\begin{tabular}{|c|c|c|c|c|c|}
\hline $\begin{array}{l}\text { Age } \\
\text { (months } \\
\text { and } \\
\text { days) }\end{array}$ & $\begin{array}{l}\text { Body } \\
\text { weight } \\
\text { (g) }\end{array}$ & $\begin{array}{l}\text { Height } \\
(\mathrm{cm})\end{array}$ & $\begin{array}{c}\text { Circumference } \\
\text { of head } \\
(\mathrm{cm})\end{array}$ & $\begin{array}{c}\text { Circumference } \\
\text { of chest } \\
(\mathrm{cm})\end{array}$ & Diet \\
\hline Birth & 2,880 & & & & \multirow{6}{*}{$\begin{array}{l}\text { Mothers' milk and } \\
\text { dried powdered milk }\end{array}$} \\
\hline $0-29$ & 4,260 & 53.3 & 36.7 & 36.0 & \\
\hline $1-27$ & 5,600 & 59.5 & 39.0 & 40.5 & \\
\hline & $(119 \%)^{*}$ & $(107 \%) *$ & $(102 \%) *$ & $(107 \%)^{*}$ & \\
\hline $2-24$ & 6,500 & 60.8 & 41.5 & 44.0 & \\
\hline $4-26$ & 8,250 & 69.2 & 43.5 & 45.0 & \\
\hline $5-24$ & 8,470 & 70.4 & 44.5 & 46.0 & \multirow{6}{*}{$\begin{array}{l}+ \text { Rice-gruel, egg-yolk, } \\
\text { fish and vegetables }\end{array}$} \\
\hline $6-28$ & 8,850 & 71.7 & 45.5 & 47.0 & \\
\hline $7-25$ & 9,100 & 73.2 & 45.5 & 45.0 & \\
\hline $8-12$ & 9,230 & 72.8 & 46.0 & 47.0 & \\
\hline $10-26$ & 9,570 & 77.2 & 46.5 & 47.0 & \\
\hline & $(111 \%)^{*}$ & $(107 \%) *$ & $(102 \%)^{*}$ & $(104 \%) *$ & \\
\hline
\end{tabular}

$(\quad)^{*}$, cf. Table 1

Note. Sitting, 6 months.; standing 10 months. 
TABLE 11. Distribution of energy $\%$ at each of frequency bands of EEG of 'healthy infants'

\begin{tabular}{|c|c|c|c|c|c|c|c|c|c|c|c|c|}
\hline \multirow{2}{*}{$\begin{array}{c}\text { Age } \\
\text { (months) }\end{array}$} & \multirow{2}{*}{ Case } & \multicolumn{11}{|c|}{ Energy $\%$} \\
\hline & & I & II & III & IV & $\mathrm{V}$ & VI & VII & VIII & IX & $\mathrm{X}$ & $\frac{\text { VIII }+\mathrm{IX}}{\mathrm{II}+\mathrm{III}}$ \\
\hline 1 & $\begin{array}{c}4 \\
10 \\
\text { Average }\end{array}$ & $\begin{array}{l}13.7 \\
19.0 \\
16.3\end{array}$ & $\begin{array}{l}12.1 \\
17.3 \\
14.7\end{array}$ & $\begin{array}{l}17.4 \\
17.3 \\
17.3\end{array}$ & $\begin{array}{l}13.7 \\
11.3 \\
12.5\end{array}$ & $\begin{array}{l}12.1 \\
10.0 \\
11.0\end{array}$ & $\begin{array}{l}9.1 \\
8.8 \\
8.9\end{array}$ & $\begin{array}{l}7.7 \\
6.6 \\
7.1\end{array}$ & $\begin{array}{l}5.4 \\
3.9 \\
4.6\end{array}$ & $\begin{array}{l}5.4 \\
3.9 \\
4.6\end{array}$ & $\begin{array}{l}3.4 \\
1.9 \\
2.6\end{array}$ & $\begin{array}{l}0.36 \\
0.22 \\
0.28\end{array}$ \\
\hline 2 & \begin{tabular}{|c|}
2 \\
4 \\
7 \\
10 \\
Average
\end{tabular} & $\begin{array}{l}16.2 \\
27.3 \\
17.6 \\
29.4 \\
22.6\end{array}$ & $\begin{array}{l}13.4 \\
17.0 \\
13.7 \\
18.0 \\
15.5\end{array}$ & $\begin{array}{l}13.4 \\
17.0 \\
17.6 \\
16.1 \\
16.0\end{array}$ & $\begin{array}{r}13.4 \\
9.2 \\
13.7 \\
8.0 \\
11.0\end{array}$ & $\begin{array}{r}13.4 \\
7.6 \\
10.3 \\
8.0 \\
9.8\end{array}$ & $\begin{array}{r}10.8 \\
7.6 \\
8.8 \\
5.6 \\
8.2\end{array}$ & $\begin{array}{l}8.6 \\
7.6 \\
7.4 \\
6.7 \\
7.5\end{array}$ & $\begin{array}{l}4.8 \\
2.7 \\
3.9 \\
2.7 \\
3.5\end{array}$ & $\begin{array}{l}4.8 \\
2.7 \\
3.9 \\
3.6 \\
3.6\end{array}$ & $\begin{array}{l}1.2 \\
1.2 \\
3.0 \\
2.0 \\
1.8\end{array}$ & $\begin{array}{l}0.35 \\
0.15 \\
0.24 \\
0.18 \\
0.22\end{array}$ \\
\hline 3 & $\begin{array}{c}6 \\
7 \\
10 \\
\text { Average }\end{array}$ & $\begin{array}{l}16.7 \\
17.8 \\
15.1 \\
16.6\end{array}$ & $\begin{array}{l}13.8 \\
14.6 \\
15.1 \\
14.4\end{array}$ & $\begin{array}{l}16.7 \\
17.8 \\
16.5 \\
17.0\end{array}$ & $\begin{array}{l}16.7 \\
13.1 \\
19.3 \\
16.3\end{array}$ & $\begin{array}{r}13.8 \\
11.7 \\
8.3 \\
11.2\end{array}$ & $\begin{array}{l}8.8 \\
7.9 \\
9.3 \\
8.6\end{array}$ & $\begin{array}{l}6.7 \\
7.9 \\
6.4 \\
7.0\end{array}$ & $\begin{array}{l}2.2 \\
3.3 \\
4.1 \\
3.2\end{array}$ & $\begin{array}{l}3.4 \\
4.0 \\
2.3 \\
3.2\end{array}$ & $\begin{array}{l}1.2 \\
2.0 \\
3.5 \\
2.2\end{array}$ & $\begin{array}{l}0.18 \\
0.22 \\
0.19 \\
0.20\end{array}$ \\
\hline 4 & \begin{tabular}{|c|}
3 \\
6 \\
7 \\
8 \\
9 \\
Average
\end{tabular} & $\begin{array}{r}17.2 \\
11.4 \\
16.1 \\
13.7 \\
8.4 \\
13.3\end{array}$ & $\begin{array}{l}17.4 \\
12.1 \\
11.7 \\
12.2 \\
12.5 \\
13.1\end{array}$ & $\begin{array}{l}17.0 \\
11.4 \\
16.2 \\
13.7 \\
12.1 \\
14.0\end{array}$ & $\begin{array}{l}13.7 \\
14.1 \\
15.9 \\
16.9 \\
13.9 \\
14.9\end{array}$ & $\begin{array}{r}9.8 \\
16.1 \\
13.6 \\
12.2 \\
13.9 \\
13.1\end{array}$ & $\begin{array}{r}7.1 \\
12.1 \\
10.2 \\
10.8 \\
15.3 \\
11.1\end{array}$ & $\begin{array}{r}6.6 \\
10.1 \\
7.1 \\
9.5 \\
12.3 \\
9.1\end{array}$ & $\begin{array}{l}4.3 \\
4.7 \\
2.2 \\
4.2 \\
5.3 \\
4.1\end{array}$ & $\begin{array}{l}3.7 \\
5.4 \\
5.1 \\
4.2 \\
3.5 \\
4.3\end{array}$ & $\begin{array}{l}3.2 \\
2.7 \\
2.0 \\
2.7 \\
2.7 \\
2.6\end{array}$ & $\begin{array}{l}0.23 \\
0.42 \\
0.26 \\
0.32 \\
0.35 \\
0.30\end{array}$ \\
\hline 5 & $\begin{array}{c}3 \\
4 \\
8 \\
10 \\
\text { Average }\end{array}$ & $\begin{array}{r}11.9 \\
9.7 \\
9.2 \\
15.0 \\
11.4\end{array}$ & $\begin{array}{r}10.4 \\
9.8 \\
9.2 \\
12.8 \\
10.0\end{array}$ & $\begin{array}{l}13.4 \\
15.0 \\
11.5 \\
16.0 \\
13.9\end{array}$ & $\begin{array}{l}18.5 \\
18.2 \\
18.3 \\
15.5 \\
17.6\end{array}$ & $\begin{array}{l}15.0 \\
14.3 \\
18.3 \\
14.2 \\
15.7\end{array}$ & $\begin{array}{l}10.4 \\
11.9 \\
11.5 \\
10.7 \\
11.1\end{array}$ & $\begin{array}{r}9.1 \\
8.8 \\
10.3 \\
7.4 \\
8.9\end{array}$ & \begin{tabular}{l|}
4.6 \\
5.4 \\
5.4 \\
3.3 \\
4.6
\end{tabular} & $\begin{array}{l}3.8 \\
4.9 \\
3.8 \\
3.1 \\
3.9\end{array}$ & $\begin{array}{l}3.0 \\
4.1 \\
2.6 \\
2.0 \\
2.9\end{array}$ & $\begin{array}{l}0.35 \\
0.41 \\
0.44 \\
0.22 \\
0.35\end{array}$ \\
\hline 6 & \begin{tabular}{|c|}
1 \\
2 \\
6 \\
7 \\
8 \\
Average
\end{tabular} & $\begin{array}{r}9.8 \\
8.6 \\
10.8 \\
10.1 \\
11.8 \\
10.2\end{array}$ & $\begin{array}{r}8.8 \\
7.9 \\
8.7 \\
10.1 \\
10.2 \\
9.1\end{array}$ & $\begin{array}{r}12.0 \\
9.4 \\
10.8 \\
11.6 \\
11.3 \\
11.0\end{array}$ & $\begin{array}{l}12.0 \\
14.0 \\
13.0 \\
16.8 \\
11.0 \\
13.3\end{array}$ & $\begin{array}{l}14.4 \\
19.4 \\
14.2 \\
16.8 \\
16.2 \\
16.2\end{array}$ & \begin{tabular}{l|}
13.2 \\
16.0 \\
13.0 \\
14.9 \\
12.6 \\
13.9
\end{tabular} & $\begin{array}{r}12.0 \\
10.3 \\
11.9 \\
8.7 \\
11.2 \\
10.8\end{array}$ & $\begin{array}{l}7.9 \\
6.4 \\
7.8 \\
4.2 \\
7.0 \\
6.6\end{array}$ & $\begin{array}{l}6.1 \\
4.6 \\
6.0 \\
4.2 \\
5.2 \\
5.2\end{array}$ & $\begin{array}{l}3.9 \\
3.5 \\
3.9 \\
2.5 \\
3.5 \\
3.4\end{array}$ & $\begin{array}{l}0.67 \\
0.63 \\
0.70 \\
0.41 \\
0.56 \\
0.58\end{array}$ \\
\hline 7 & \begin{tabular}{|c|}
2 \\
4 \\
6 \\
8 \\
10 \\
Average
\end{tabular} & $\begin{array}{r}15.5 \\
7.7 \\
10.2 \\
10.0 \\
10.1 \\
10.7\end{array}$ & $\begin{array}{l}7.7 \\
6.2 \\
8.5 \\
6.5 \\
9.7 \\
7.7\end{array}$ & $\begin{array}{r}7.8 \\
11.1 \\
10.6 \\
8.3 \\
10.6 \\
9.6\end{array}$ & $\begin{array}{l}12.4 \\
16.6 \\
10.5 \\
15.7 \\
17.8 \\
14.6\end{array}$ & $\begin{array}{l}21.4 \\
14.7 \\
10.7 \\
25.0 \\
19.0 \\
18.1\end{array}$ & $\begin{array}{l}11.9 \\
14.0 \\
14.0 \\
13.6 \\
11.8 \\
13.0\end{array}$ & $\begin{array}{r}9.7 \\
16.0 \\
15.2 \\
8.8 \\
9.3 \\
11.8\end{array}$ & $\begin{array}{l}6.1 \\
6.9 \\
9.8 \\
5.8 \\
6.3 \\
6.9\end{array}$ & $\begin{array}{l}4.4 \\
4.0 \\
6.8 \\
4.0 \\
3.1 \\
4.4\end{array}$ & $\begin{array}{l}3.0 \\
2.7 \\
3.7 \\
2.4 \\
2.1 \\
2.7\end{array}$ & $\begin{array}{l}0.67 \\
0.63 \\
0.86 \\
0.66 \\
0.46 \\
0.65\end{array}$ \\
\hline 8 & \begin{tabular}{|c|}
4 \\
5 \\
6 \\
7 \\
8 \\
10 \\
Average
\end{tabular} & $\begin{array}{r}8.6 \\
7.9 \\
11.2 \\
7.9 \\
8.2 \\
8.9 \\
8.7\end{array}$ & $\begin{array}{l}6.1 \\
7.0 \\
8.0 \\
7.4 \\
7.6 \\
8.7 \\
7.4\end{array}$ & $\begin{array}{r}9.3 \\
8.3 \\
9.0 \\
8.0 \\
9.6 \\
10.3 \\
9.0\end{array}$ & $\begin{array}{r}13.2 \\
9.4 \\
11.2 \\
8.4 \\
12.5 \\
13.1 \\
11.3\end{array}$ & $\begin{array}{l}16.8 \\
14.9 \\
16.1 \\
13.3 \\
18.5 \\
22.3 \\
16.9\end{array}$ & $\begin{array}{l}13.5 \\
18.6 \\
14.3 \\
12.6 \\
14.1 \\
14.4 \\
14.5\end{array}$ & $\begin{array}{r}20.7 \\
15.3 \\
13.8 \\
22.6 \\
12.5 \\
9.5 \\
15.7\end{array}$ & $\begin{array}{r}7.5 \\
9.9 \\
6.9 \\
12.6 \\
8.2 \\
6.6 \\
8.6\end{array}$ & $\begin{array}{l}1.8 \\
4.4 \\
5.7 \\
3.1 \\
4.4 \\
3.0 \\
3.7\end{array}$ & $\begin{array}{l}2.5 \\
4.3 \\
3.8 \\
4.2 \\
4.4 \\
3.3 \\
3.7\end{array}$ & $\begin{array}{l}0.60 \\
0.93 \\
0.74 \\
1.01 \\
0.73 \\
0.50 \\
0.75\end{array}$ \\
\hline
\end{tabular}


TABLE 11. Continued

\begin{tabular}{|c|c|c|c|c|c|c|c|c|c|c|c|c|}
\hline \multirow{2}{*}{$\begin{array}{c}\text { Age } \\
\text { (months) }\end{array}$} & \multirow{2}{*}{ Cases } & \multicolumn{11}{|c|}{ Energy \% } \\
\hline & & $\mathbf{I}$ & II & III & IV & $\mathrm{V}$ & VI & VII & VIII & IX & $X$ & $\begin{array}{l}\text { VIII +IX } \\
\text { II + IIII }\end{array}$ \\
\hline 9 & $\begin{array}{c}1 \\
2 \\
7 \\
10 \\
\text { Average }\end{array}$ & $\begin{array}{l}11.3 \\
12.6 \\
11.2 \\
11.7 \\
11.7\end{array}$ & \begin{tabular}{r|}
9.8 \\
8.1 \\
10.6 \\
8.7 \\
9.3
\end{tabular} & $\begin{array}{r}9.6 \\
9.2 \\
10.6 \\
9.0 \\
9.6\end{array}$ & $\begin{array}{r}9.7 \\
12.6 \\
10.3 \\
11.9 \\
11.1\end{array}$ & $\begin{array}{l}10.7 \\
22.1 \\
12.4 \\
17.9 \\
15.7\end{array}$ & $\begin{array}{l}11.7 \\
12.0 \\
12.2 \\
14.5 \\
12.6\end{array}$ & $\begin{array}{l}14.8 \\
10.0 \\
13.1 \\
12.9 \\
12.7\end{array}$ & $\begin{array}{l}9.5 \\
7.1 \\
9.8 \\
7.0 \\
8.3\end{array}$ & $\begin{array}{l}8.1 \\
3.2 \\
4.9 \\
3.5 \\
4.9\end{array}$ & $\begin{array}{l}4.8 \\
3.0 \\
5.1 \\
3.0 \\
3.9\end{array}$ & $\begin{array}{l}0.90 \\
0.59 \\
0.69 \\
0.60 \\
0.69\end{array}$ \\
\hline 10 & $\begin{array}{c}2 \\
4 \\
6 \\
7 \\
\text { Average }\end{array}$ & $\begin{array}{r}12.3 \\
8.7 \\
8.1 \\
9.6 \\
9.6\end{array}$ & $\begin{array}{l}9.4 \\
8.7 \\
7.3 \\
6.9 \\
8.0\end{array}$ & $\begin{array}{r}10.3 \\
6.8 \\
8.9 \\
9.6 \\
8.9\end{array}$ & $\begin{array}{r}7.7 \\
10.7 \\
10.7 \\
12.8 \\
10.4\end{array}$ & $\begin{array}{l}11.3 \\
15.4 \\
18.3 \\
14.5 \\
14.8\end{array}$ & $\begin{array}{l}15.6 \\
15.4 \\
17.1 \\
14.5 \\
15.6\end{array}$ & \begin{tabular}{l|}
13.3 \\
18.1 \\
13.7 \\
12.8 \\
14.4
\end{tabular} & $\begin{array}{r}10.3 \\
8.7 \\
8.9 \\
11.1 \\
9.7\end{array}$ & $\begin{array}{l}4.8 \\
3.8 \\
3.0 \\
3.6 \\
3.8\end{array}$ & $\begin{array}{l}4.8 \\
3.8 \\
4.0 \\
4.6 \\
4.3\end{array}$ & $\begin{array}{l}0.76 \\
0.80 \\
0.73 \\
0.89 \\
0.79\end{array}$ \\
\hline 11 & $\begin{array}{c}1 \\
2 \\
10 \\
\text { Average }\end{array}$ & $\begin{array}{r}7.1 \\
13.8 \\
10.7 \\
10.5\end{array}$ & $\begin{array}{r}7.1 \\
10.2 \\
7.9 \\
8.4\end{array}$ & $\begin{array}{l}7.9 \\
8.2 \\
8.3 \\
8.1\end{array}$ & $\begin{array}{r}9.6 \\
11.9 \\
15.0 \\
12.1\end{array}$ & $\begin{array}{l}12.5 \\
10.2 \\
16.8 \\
13.1\end{array}$ & $\begin{array}{l}12.9 \\
12.8 \\
12.7 \\
12.8\end{array}$ & $\begin{array}{l}14.0 \\
13.8 \\
12.2 \\
13.3\end{array}$ & $\begin{array}{r}14.5 \\
10.2 \\
8.8 \\
11.1\end{array}$ & $\begin{array}{l}7.2 \\
5.5 \\
4.5 \\
5.7\end{array}$ & $\begin{array}{l}7.3 \\
3.3 \\
3.1 \\
4.5\end{array}$ & $\begin{array}{l}1.44 \\
0.85 \\
0.82 \\
1.01\end{array}$ \\
\hline 12 & $\begin{array}{c}2 \\
4 \\
6 \\
\text { Average }\end{array}$ & $\begin{array}{r}12.7 \\
8.7 \\
7.6 \\
9.6\end{array}$ & $\begin{array}{l}7.6 \\
6.2 \\
6.8 \\
6.8\end{array}$ & $\begin{array}{l}7.3 \\
7.5 \\
9.4 \\
8.0\end{array}$ & $\begin{array}{l}19.8 \\
12.6 \\
15.5 \\
15.9\end{array}$ & $\begin{array}{l}12.3 \\
15.2 \\
17.0 \\
14.8\end{array}$ & $\begin{array}{r}9.6 \\
13.8 \\
13.8 \\
12.4\end{array}$ & $\begin{array}{l}11.3 \\
13.5 \\
13.2 \\
12.6\end{array}$ & $\begin{array}{r}10.0 \\
12.4 \\
9.7 \\
10.7\end{array}$ & $\begin{array}{l}5.9 \\
6.5 \\
4.4 \\
5.6\end{array}$ & $\begin{array}{l}3.6 \\
3.7 \\
3.0 \\
3.4\end{array}$ & $\begin{array}{l}1.06 \\
1.37 \\
0.87 \\
1.10\end{array}$ \\
\hline
\end{tabular}

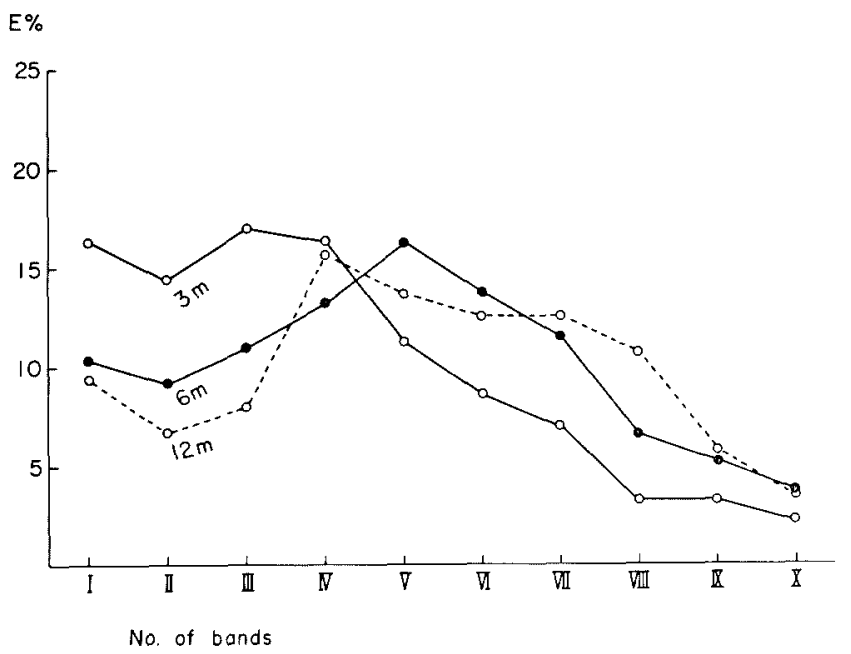

Fig. 1. Distribution of the energy $\%(\mathrm{E} \%)$ at each of frequency bands of the EEG basic waves of healthy infants.

with the mean values reported in 1960 from the Department of Welfare of Japan.

The circumference of the head was found to be $99-100 \%$ of the standard values. 


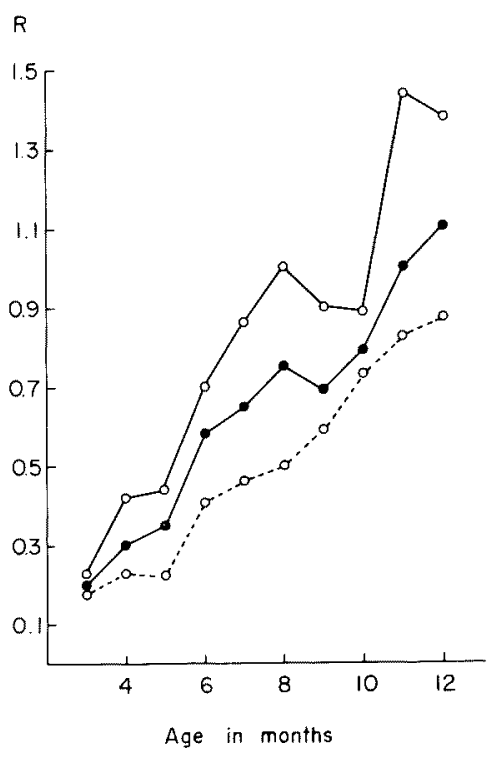

Fig. 2. Maturation pattern of the EEG basic waves in healthy infants expressed by maximum, average and minimum values of ' $R$ '.

$$
' \mathrm{R} '=\frac{\mathrm{E} \% \text { of } \mathrm{VIII}+\mathrm{E} \% \text { of IX }}{\mathrm{E} \% \text { of } \mathrm{II}+\mathrm{E} \% \text { of III }}
$$

Changes in the frequency pattern of the EEG basic waves of healthy infants with advance in age

Distribution patterns of $\mathrm{E} \%$ at each of frequency bands of the EEG basic waves of the infants were shown in Table 11. Fig. 1 was drawn from the average values of $\mathrm{E} \%$ at each of frequency bands of infants aged 3,6 and 12 months, showing that there was a tendency to a decrease in $\mathrm{E} \%$ at the II and III bands with a concomitant increase in that at the VIII and IX bands (cf. Fig. 1).

These changes in the distribution of $\mathrm{E} \%$ at each of frequency bands according to the age of infants were more clearly demonstrated when ' $\mathrm{R}$ ' $=(\mathrm{E} \%$ of VIII $+\mathrm{E} \%$ of $\mathrm{IX}) /(\mathrm{E} \%$ of $\mathrm{II}+\mathrm{E} \%$ of III) was calculated and plotted against the age in months (Fig. 2), which might be used as a simplified figure for an evaluation of a maturation pattern of the EEG basic waves of healthy infants.

\section{References}

1) Arakawa, Ts., Mizuno, T., Honda, Y., Tamura, T., Sakai, K., Tatsumi, S., Chiba, F. \& Coursin, D.B. (a) Brain function of infants fed on milk from mothers with low serum folate levels. Tohoku J. exp. Med., 1969, 97, 391-397.

2) Arakawa, Ts., Mizuno, T., Sakai, K., Chida, N., Watanabe, A., Ohara, K. \& Coursin, D.B. (b) Electroencephalographic frequency patterns of rats treated with aminopterin in early infancy. Tohoku J. exp. Med., 1969, 97, 385-390. 